\title{
Pulmonary Lymphangitic Carcinomatosis due to Renal Cell Carcinoma
}

\author{
Achuta K. Guddati $^{a} \quad$ Creticus P. Marak $^{b}$ \\ ${ }^{a}$ Department of Internal Medicine, St. Vincent's Hospital, Bridgeport, Conn., \\ and ${ }^{b}$ Division of Pulmonary and Critical Care Medicine, Montefiore Hospital, \\ Albert Einstein College of Medicine, New York, N.Y., USA
}

\section{Key Words}

Pulmonary lymphangitic carcinomatosis $\cdot$ Renal cell carcinoma

\begin{abstract}
Renal cell carcinoma is an aggressive disease with a high rate of mortality. It is known to metastasize to the lung, liver, bone and brain. However, manifestation through lymphatic spread to the lungs is rare. Lymphangitic carcinomatosis is commonly observed in malignancies of the breast, lung, pancreas, colon and cervix. It is unusual to observe lymphangitic carcinomatosis of the lungs due to renal cell carcinoma. Lymphangitic carcinomatosis of the lungs may result in severe respiratory distress and may be the direct cause of death. Currently, there are no known modalities of preventing or slowing lymphangitic carcinomatosis besides treating the primary tumor. However, early detection may change the course of the disease and may prolong survival. This is compounded by the difficulty involved in diagnosing lymphangitic carcinomatosis of the lung which frequently involves lung biopsy. Immunohistochemical studies are often used in conjunction with regular histochemistry in ascertaining the primary tumor and in differentiating it from pulmonary metastasis. In this case report, we describe the presentation and clinical course of renal cell carcinoma in a patient which manifested as lymphangitis carcinomatosa of the lungs. The patient underwent surgical resection of the primary tumor with lymph node resection but presented with a fulminant lymphangitic carcinomatosis of the lungs within two weeks. Immunohistochemistry of the tissue obtained by the biopsy confirmed the diagnosis which was subsequently corroborated during his autopsy. This case illustrates the necessity of an urgent follow-up of chemotherapy and immunotherapy in such patients.
\end{abstract}

Dr. Achuta K. Guddati, MD, PhD 


\section{Introduction}

Renal cell carcinoma originates in the renal cortex and usually presents at an advanced stage with a $50 \%$ mortality rate after 5 years of diagnosis [1]. It classically presents as hematuria, flank pain and abdominal mass [2] but may also manifest with a variety of paraneoplastic syndromes including anemia, hypercalcemia, erythrocytosis, amyloidosis and thrombocytosis [3]. Common sites of metastasis are lung, bone, liver and brain. Notably, more than $90 \%$ of metastases in the lung have been detected by chest X-ray (CXR) in asymptomatic patients [4].

Pulmonary embolism involves the establishment of the tumor in the pulmonary vasculature, but renal cell carcinoma also frequently invades the lymphatic system. Isolated lymph node metastases due to renal cell carcinoma are infrequent but have been reported in medical literature [5,6]. Spread beyond the lymph nodes may occur through the lymphatic system, resulting in lymphangitic carcinomatosis. Lymphangitis carcinomatosa of the lungs is distinct from pulmonary metastases as the tumor emboli do not proliferate or spread locally. They are often seen in collections measuring less than 10 microns and frequently trigger the coagulation cascade and obstruct the flow of blood in pulmonary capillaries. This may result in severe respiratory distress and often becomes the direct cause of death. This case report details the presentation and clinical course of a patient with renal cell carcinoma who was found to have lymphangitic carcinomatosis which rapidly progressed in a time span of two weeks resulting in his demise.

\section{Case Report}

A 49-year-old man with hypertension presented with left flank pain. He denied night sweats and weight loss, and was a lifelong non-smoker. MRI showed a left renal mass with extensive retroperitoneal lymph node involvement. He underwent radical nephrectomy with retroperitoneal lymph node dissection. Hilar lymph nodes (3/3), para-aortic lymph nodes (8/8) and interaortocaval lymph nodes $(7 / 7)$ tested positive for tumor involvement. The left kidney was found to have a $9.8-\mathrm{cm}$ mass at the lower pole and biopsy showed clear cell type renal cell carcinoma with focal sarcomatoid and rhabdoid cells. Fig. 1a shows high-grade spindle cells in the sarcomatoid focus and fig. $1 \mathrm{~b}$ shows rhabdoid cells with large eccentric nuclei and abundant cytoplasm. The adrenal gland was also found to be infiltrated with tumor.

He was readmitted two weeks later with right flank pain, dyspnea, fever and cough productive of yellowish sputum. Physical examination was significant for tachycardia, tachypnea, bilateral crepitations in the chest and bilateral 1+ pedal edema. His arterial blood gas showed respiratory alkalosis with an elevated alveolar-arterial (A-a) gradient of 52. His CBC showed leukocytosis at 19,800 cells/ $\mu \mathrm{l}$ with $88 \%$ neutrophils, hemoglobin of $9.8 \mathrm{~g} / \mathrm{dl}$ and a platelet count of $588 \mathrm{k} / \mu \mathrm{l}$. His LFTs were deranged with an albumin of $2.5 \mathrm{~g} / \mathrm{dl}$, ALK of 315 and AST/ALT of 166/184. His ESR was elevated at 134 and BUN/creatinine rapidly rose from 13/1 to 24/1.9. Notably, his CXR showed marked infiltration with nodular opacities in all lung fields bilaterally. Fig. 2a shows his CXR during his first admission and fig. $2 \mathrm{~b}$ shows his CXR at the second admission which reveals marked changes compared to his initial CXR.

He was started on Zosyn and vancomycin and was later switched to aztreonam and clindamycin on account of worsening renal function. His respiratory and blood cultures were negative for any growth. He continued to be febrile, hypoxemic and tachycardic. CT scan to rule out pulmonary embolism could not be performed due to renal failure and Doppler ultrasound of the lower extremities was negative for DVT. CT scan without contrast showed nodular opacities with extensive septal thickening as depicted in fig. 3 . An echocardiogram showed a hyperdynamic left ventricle with EF of $80 \%$. The right ventricle size and function were normal. Bronchoscopy with transbronchial biopsy of the right medial 
lobe was done and biopsy samples revealed non-small cell cancer with clear cell features consistent with renal cell carcinoma and lymphangectatic invasion. Immunohistochemistry showed that the tissue stained positively for vimentin, CD10 and CD 31 as depicted in fig. 4 , confirming the renal origin of his tumor. His renal function worsened and he was transferred to the medical ICU where he developed septic shock, he was made DNR and passed away shortly thereafter. The autopsy report showed florid lymphangectatic invasion with focal necrosis, affecting all lobes of both lungs.

\section{Discussion}

Lymphangitic spread of metastatic cancer has been observed in many malignancies including breast, lung, pancreatic, colonic and cervical cancers [7, 8]. Symptoms of tachycardia, tachypnea, hypoxemia and clear lung fields in a patient with malignancy should raise the suspicion of lymphangitic carcinomatosis. Infiltration of the tumor cells into the superficial lymphatic system can be readily detected as lymphedema in some malignancies, especially breast cancer. This may even be the initial manifestation of a malignancy [9] or may portend massive tumor burden due to an internal malignancy [10]. Histologically, this is evidenced by extensive lymphatic invasion by cords of tumor cells. The patient may suffer from intense pain, ulceration and necrosis if the superficial lymphatic system is affected as against a painless gradual course when the deeper lymphatics are involved. Fever and leukocytosis often accompany lymphangitic carcinomatosis making an effective diagnosis difficult. Ventilation perfusion scans may be normal or may show a mottled appearance with multiple small, peripheral, subsegmental perfusion defects with normal ventilation. This pattern can be readily distinguished from the pattern seen with thromboemboli. CT angiography may show dilated and beaded peripheral pulmonary arteries. Pulmonary angiography is insensitive and may show subtle defects including subsegmental filling defects, pruning and tortuosity of the third- to fifth-order vessels, or delayed filling of segmental arteries. It may however help in ruling out larger thromboemboli. FDG-PET similarly may detect large emboli with avid uptake but may not be sensitive to detect smaller collections of tumor cells.

The gold standard of diagnosis is lung biopsy with demonstration of microscopic emboli of tumor cells in the pulmonary vasculature and detection of circulating tumor cells in the blood drawn from the pulmonary artery catheter. However, it should be noted that lymphangitic carcinomatosis may not necessarily be blood borne. Pulmonary lymphatics communicate with the lymphatic system of the neck and direct extension of head and neck cancers has been observed to occur through this route [11]. Lymphangitic carcinomatosis may also present in an insidious manner being the first sign of an occult malignancy. It has also been noted to be asymptomatic with the only finding being an abnormal CXR [12]. The presence of vimentin and CD10 noted on immunohistochemistry confirmed the diagnosis of clear cell renal cell carcinoma in this patient. It is interesting to note that the tissue samples of this patient were positive for CD31 which inversely correlates with TNM staging and nuclear grade [13]. The sarcomatoid features noted in some samples excised during his initial surgery may explain the aggressive course of his disease as it has been shown that sarcomatoid renal cell carcinoma is a rapidly progressive disease with poor overall survival $[14,15]$.

This case report demonstrates the rapid clinical deterioration a patient may experience due to pulmonary lymphangitic carcinomatosis. It is notable that this 
patient underwent successful resection but developed lymphangitic carcinomatosis within two weeks which likely was the direct cause of his death. Pulmonary lymphangitic carcinomatosis has a poor prognosis especially since it denotes systemic metastasis. Early detection with the help of imaging mediated by tumor-specific antibodies can result in therapeutic interventions which may slow the progression of the disease or may even be life-saving.

\section{Disclosure Statement}

The authors have no conflicts of interest to disclose.

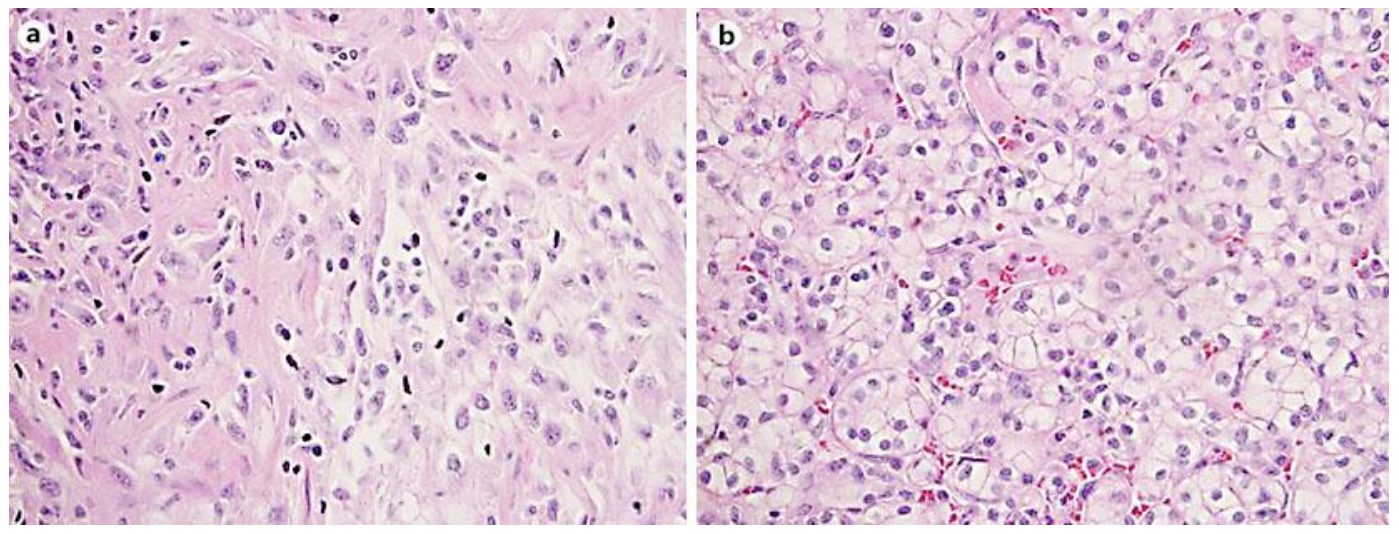

Fig. 1. Hematoxylin and eosin staining of tumor sample. a High-grade spindle cells in sarcomatoid $\overline{\text { focus. }} \mathbf{b}$ Rhabdoid cells with large eccentric nuclei and abundant eosinophilic cytoplasm. 

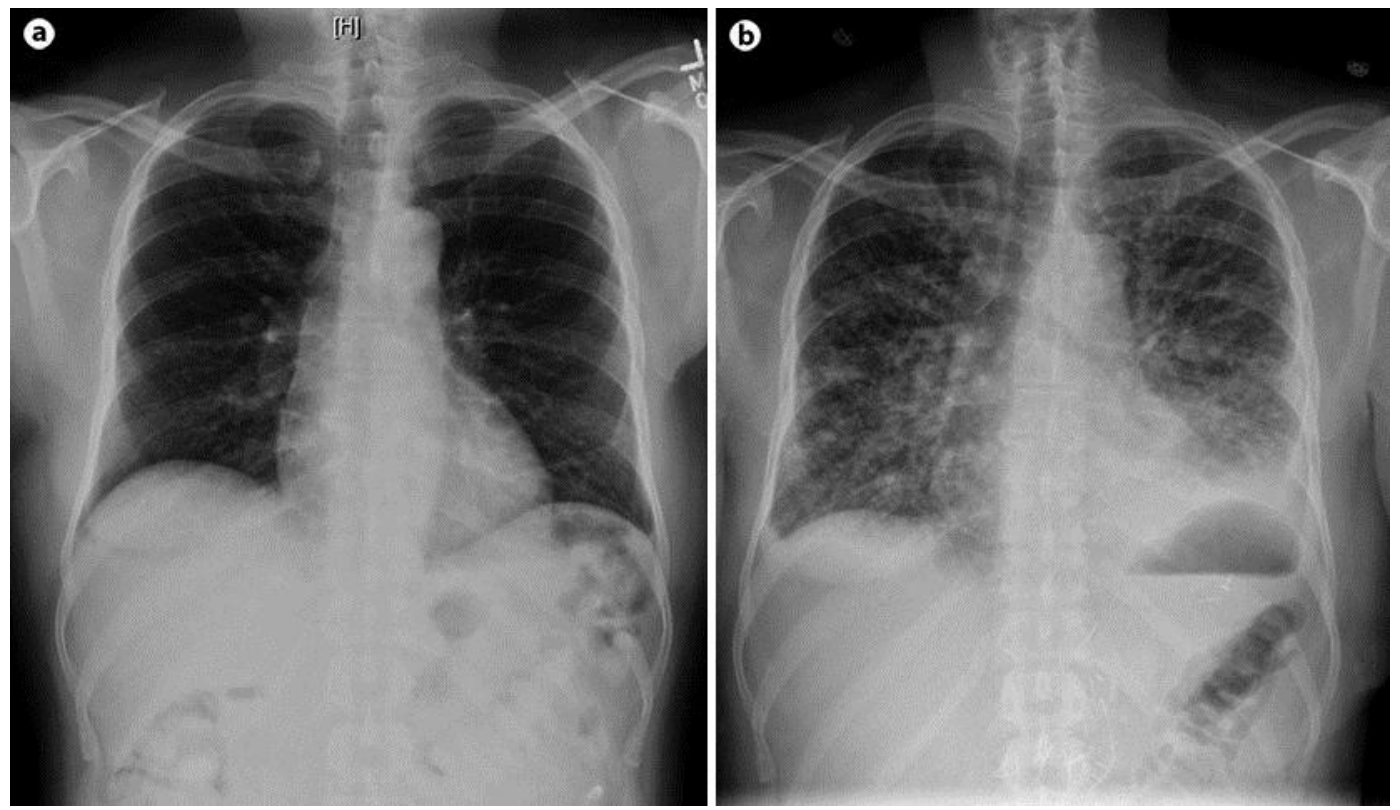

Fig. 2. CXR depicting the interval change between the first and the second admission. a CXR on first admission with no signs of pulmonary involvement. $\mathbf{b}$ CXR on second admission depicting reticulo-nodular involvement in all lung fields.

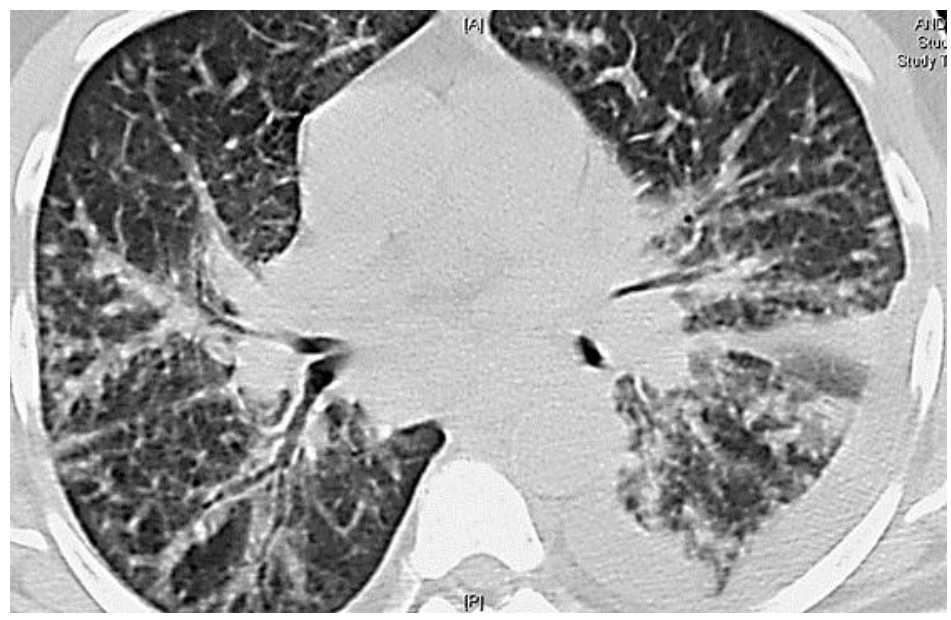

Fig. 3. Axial CT scan depicting extensive involvement of the parenchyma with septal thickening. 

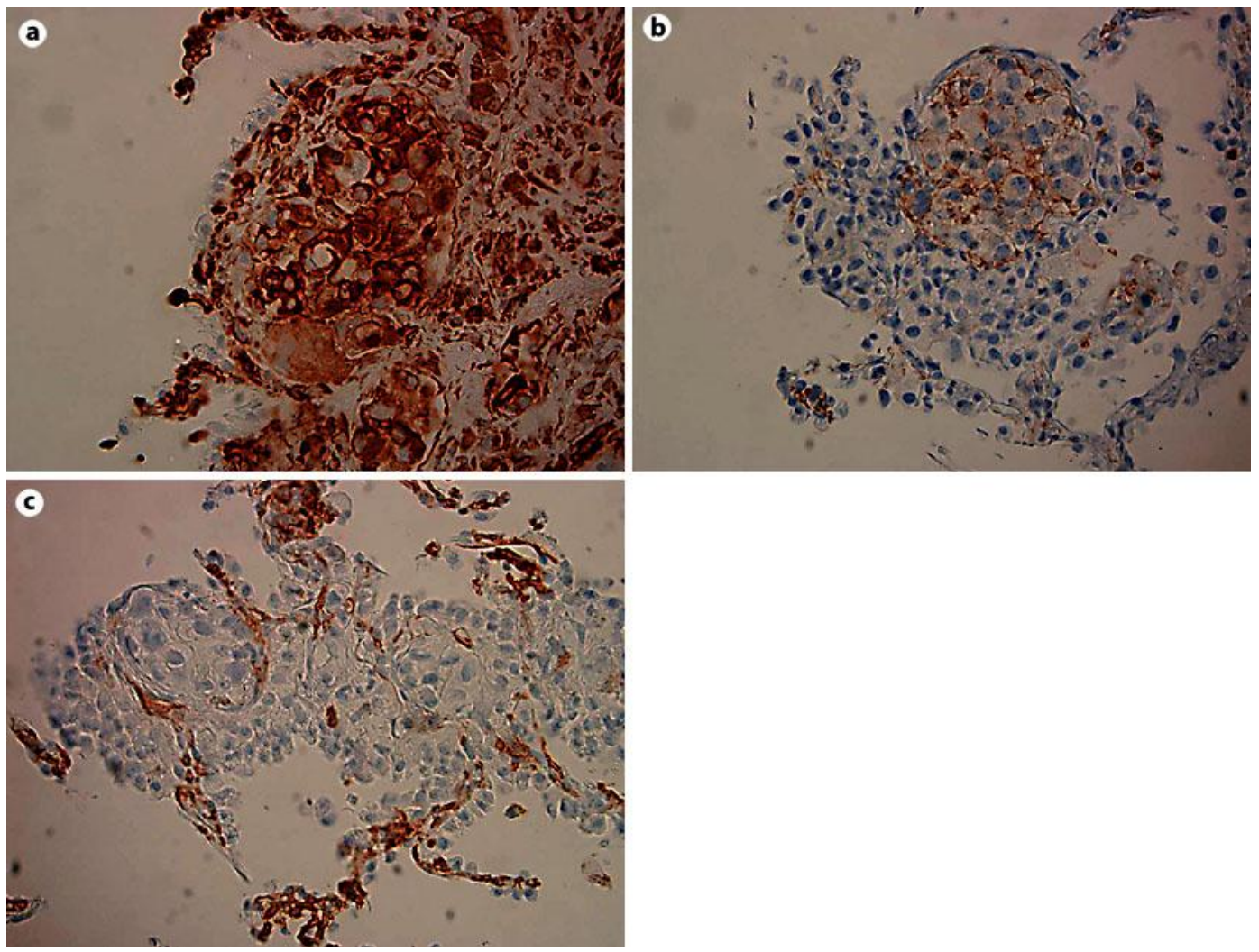

Fig. 4. Immunohistochemistry of sample obtained from lung biopsy. a Immunostaining for vimentin. b Immunostaining for CD10. c Immunostaining for CD31. 


\section{References}

1 Cho E, Adami HO, Lindblad P: Epidemiology of renal cell cancer. Hematol Oncol Clin North Am 2011;25:651-665.

-2 Skinner DG, Colvin RB, Vermillion CD, Pfister RC, Leadbetter WF: Diagnosis and management of renal cell carcinoma. A clinical and pathologic study of 309 cases. Cancer 1971;28:1165-1177.

-3 Gold PJ, Fefer A, Thompson JA: Paraneoplastic manifestations of renal cell carcinoma. Semin Urol Oncol 1996;14:216-222.

-4 Hafez KS, Novick AC, Campbell SC: Patterns of tumor recurrence and guidelines for followup after nephron sparing surgery for sporadic renal cell carcinoma. J Urol 1997;157:2067-2070.

5 Giuliani A, Caporale A, Borghese M, Galati G, Di Bari M, Demoro M: Papillary renal cell carcinoma presenting as nodal metastases to the neck. J Exp Clin Cancer Res 1999;18:579-582.

-6 Kanamaru H, Mori H, Sasaki M, et al: Histologic characteristics of renal cell carcinomas with lymph node metastasis. Int J Urol 1997;4:451-455.

7 Janower ML, Blennerhassett JB: Lymphangitic spread of metastatic cancer to the lung. A radiologic-pathologic classification. Radiology 1971;101:267-273.

-8 Bruce DM, Heys SD, Eremin 0: Lymphangitis carcinomatosa: a literature review. J R Coll Surg Edinb 1996;41:7-13.

-9 Keohane SG, Doherty VR, McLaren KM, Hunter JA: Cutaneous lymphangitis carcinomatosa: a rare primary manifestation of malignancy. J R Soc Med 1996;89:582-583.

10 Lookingbill DP, Spangler N, Sexton FM: Skin involvement as the presenting sign of internal carcinoma. A retrospective study of 7316 cancer patients. J Am Acad Dermatol 1990;22:19-26.

11 Lindqvist C, Lepantalo M, Jungell P: Lymphangitis carcinomatosa of the lungs. An unusual complication of oral cancer. Br J Oral Maxillofac Surg 1988;26:228-231.

12 Gupta PR, Joshi N, Meena RC, Ali M: Asymptomatic lymphangitis carcinomatosis due to squamous cell lung carcinoma. Indian J Chest Dis Allied Sci 2005;47:121-123.

13 Sandlund J, Hedberg Y, Bergh A, Grankvist K, Ljungberg B, Rasmuson T: Evaluation of CD31 (PECAM-1) expression using tissue microarray in patients with renal cell carcinoma. Tumour Biol 2007;28:158-164.

14 Ro JY, Ayala AG, Sella A, Samuels ML, Swanson DA: Sarcomatoid renal cell carcinoma: clinicopathologic. A study of 42 cases. Cancer 1987;59:516-526.

15 Cangiano T, Liao J, Naitoh J, Dorey F, Figlin R, Belldegrun A: Sarcomatoid renal cell carcinoma: biologic behavior, prognosis, and response to combined surgical resection and immunotherapy. J Clin Oncol 1999;17:523-528.

This study was performed at Montefiore Hospital. 